\title{
BMJ Open Deep learning-based facial image analysis in medical research: a systematic review protocol
}

\author{
Zhaohui Su (D) , ${ }^{1}$ Bin Liang, ${ }^{2}$ Feng Shi, ${ }^{3} \mathrm{~J}$ Gelfond, ${ }^{4}$ Sabina Šegalo (i) , \\ Jing Wang, ${ }^{6}$ Peng Jia, ${ }^{7,8}$ Xiaoning $\mathrm{Hao}^{9}$
}

To cite: Su Z, Liang B, Shi F, et al. Deep learning-based facial image analysis in medical research: a systematic review protocol. BMJ Open 2021;11:e047549. doi:10.1136/ bmjopen-2020-047549

- Prepublication history for this paper is available online. To view these files, please visit the journal online (http://dx.doi. org/10.1136/bmjopen-2020047549).

Received 01 December 2020 Accepted 18 August 2021

Check for updates

(C) Author(s) (or their employer(s)) 2021. Re-use permitted under CC BY-NC. No commercial re-use. See rights and permissions. Published by BMJ.

For numbered affiliations see end of article.

Correspondence to

Dr Xiaoning Hao; haoxn@nhei.cn

\section{ABSTRACT}

Introduction Deep learning techniques are gaining momentum in medical research. Evidence shows that deep learning has advantages over humans in image identification and classification, such as facial image analysis in detecting people's medical conditions. While positive findings are available, little is known about the state-of-the-art of deep learning-based facial image analysis in the medical context. For the consideration of patients' welfare and the development of the practice, a timely understanding of the challenges and opportunities faced by research on deep-learning-based facial image analysis is needed. To address this gap, we aim to conduct a systematic review to identify the characteristics and effects of deep learning-based facial image analysis in medical research. Insights gained from this systematic review will provide a much-needed understanding of the characteristics, challenges, as well as opportunities in deep learning-based facial image analysis applied in the contexts of disease detection, diagnosis and prognosis. Methods Databases including PubMed, PsycINF0, CINAHL, IEEEXplore and Scopus will be searched for relevant studies published in English in September, 2021. Titles, abstracts and full-text articles will be screened to identify eligible articles. A manual search of the reference lists of the included articles will also be conducted. The Preferred Reporting Items for Systematic Reviews and Meta-Analyses framework was adopted to guide the systematic review process. Two reviewers will independently examine the citations and select studies for inclusion. Discrepancies will be resolved by group discussions till a consensus is reached. Data will be extracted based on the research objective and selection criteria adopted in this study.

Ethics and dissemination As the study is a protocol for a systematic review, ethical approval is not required. The study findings will be disseminated via peer-reviewed publications and conference presentations.

PROSPERO registration number CRD42020196473.

\section{BACKGROUND}

As disease manifestations often show in various places in the human body, such as Down syndromes can change patients' facial features, researchers have been investigating whether analysing appearance features can facilitate early disease detection

\section{Strengths and limitations of this study}

This systematic review protocol follows the Preferred Reporting Items for Systematic Review and MetaAnalysis Protocols guidelines.

- By examining the characteristics and effects of deep learning-based facial image analysis in medical research, this systematic review bridges the gap in the literature.

- This review is limited to evidence on the use and application of deep learning technologies in patients facial image identification and classification.

- Non-English databases will not be searched, which might limit the representativeness of the results.

and identification. ${ }^{1-5}$ One promising field is deep learning-based facial analysis. ${ }^{6-8}$ Deep learning represents a powerful range of artificial intelligence (AI) algorithm that allows computers to tackle complex problems via capitalising on neural networks, such as convolutional neural networks (CNNs), that are rich in neurons, layers and interconnectivity (see figure 1 ) ${ }^{9}$ Simply put, deep learning is a mechanism that allows computers to solve complex problems by neural network architecture. This ability to develop complex network structures gives deep learning a distinctive advantage: it can automatically transform raw data input into meaningful features that enable pattern identification. ${ }^{10}$ Deep learning technique has revolutionary potential in practical and research fields. ${ }^{11}$ In practice, as deep learning effectively identifies objects, traffic signs and faces, its adaptations have been widely applied in designing robots and self-driving cars. ${ }^{12-15}$ Deep learning has also been widely adopted in biomedical and clinical research, particularly in the field of medical imaging. ${ }^{16-19}$

Medical conditions are often diagnosed by means of tests, such as biopsy and diagnostic imaging. An example list of diseases that have been analysed by deep learning technologies 


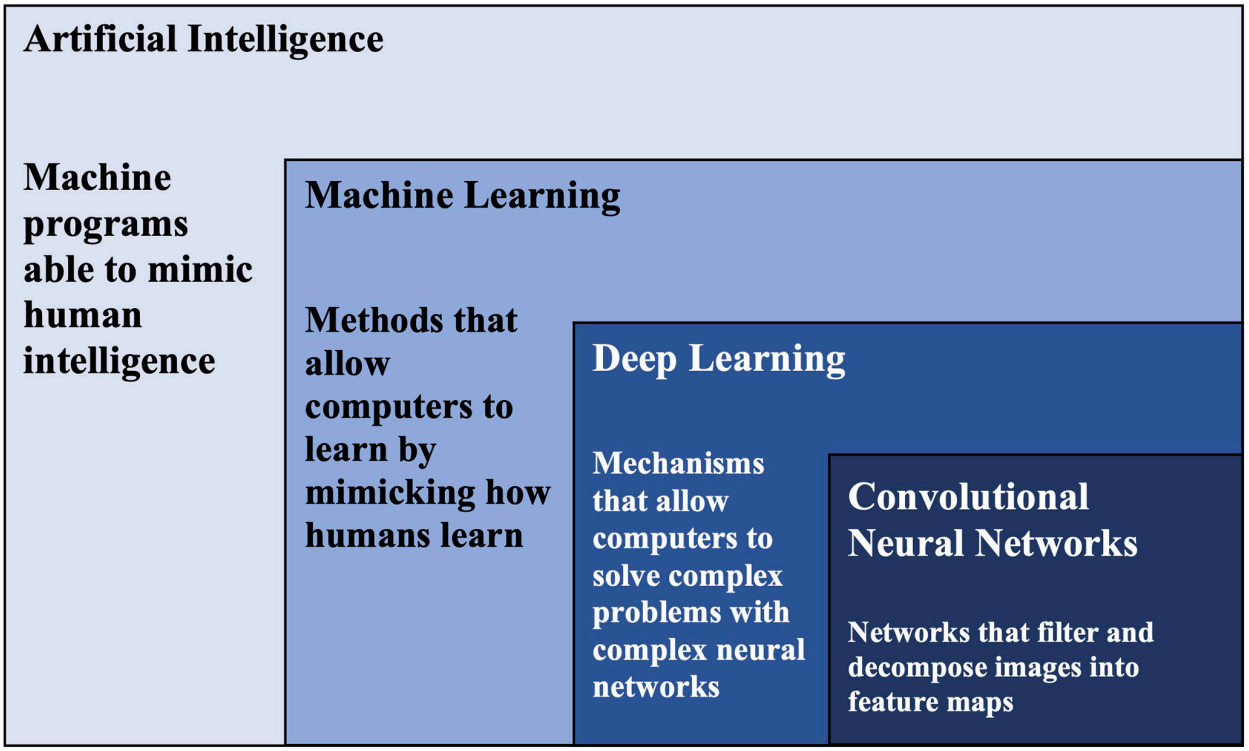

Figure 1 Relationship between artificial intelligence, machine learning, deep learning and convolutional neural networks.

could be found in table 1. As diagnostic imaging is noninvasive and can facilitate personalised medicine, it is a preferred test option for patients and healthcare practitioners. ${ }^{2021}$ This, in turn, has contributed to the exponential growth of medical imaging data and the increasing need for boosting medical image processing power to formulate diagnosis swiftly. ${ }^{21} 22$ Compared with traditional computer aided diagnosis for analysing medical imaging, such as hand-crafted radiomics for tumour detection, deep learning methods are superior in their ability to process large quantities of medical images accurately and cost-effectively, without exerting a heavy workload on radiologists. ${ }^{23-27}$ Evidence shows that deep learning-based medical image analysis was able to increase accuracy rates in various disease contexts, such as the identification of spinal disorder ${ }^{1}$ and lung cancer histology, ${ }^{28}$ classification of skin lesion ${ }^{29}$ and chronic gastritis,${ }^{30}$ and the prediction of tumour-related genes ${ }^{31}$ and vascular diseases. ${ }^{32}$
Applying the deep learning technique to perform facial recognition and analysis tasks, researchers found that the technique yielded superior results in identifying and classifying faces of people with cancer from those without. ${ }^{6}$ Similarly, examining facial phenotypes of people with genetic disorders, findings indicate that the technique was effective and was able to yield an optimal $91 \%$ top- 10 accuracy. ${ }^{33}$ Evidence further indicates that, for some tasks involving identifying and classifying facial images, deep learning techniques have often performed on par or better than human beings. ${ }^{5} 710$ 34-36 Comparing clinical and deep learning evaluations of microdeletion syndrome facial phenotypes, researchers found that deep learning outperformed clinical evaluations in terms of sensitivity and specificity by $96 \%{ }^{35}$ These findings combined suggest that deep learning-based facial analysis technology has great potential to address complex medical challenges prevalent in healthcare. However, there has not been any

Table 1 An example list of diseases that have been analysed by deep learning techniques

\begin{tabular}{|c|c|}
\hline Disease context & Deep learning technique \\
\hline Acromegaly & $\begin{array}{l}\text { Convolutional neural network (along with Generalized Linear Models; } \\
\text { K-nearest neighbors; Support Vector Machines; forests of randomized } \\
\text { trees) }\end{array}$ \\
\hline Cancer & Convolutional neutral network ${ }^{64}$ \\
\hline Cornelia de Lange syndrome & DeepGestalt technology ${ }^{7}$ \\
\hline Coronary artery disease & Convolutional neural network ${ }^{65}$ \\
\hline Facial dermatological disorders & Convolutional neural network ${ }^{67}$ \\
\hline Keratinocytic skin cancer & Convolutional neutral network ${ }^{68}$ \\
\hline Inherited retinal degenerations & Convolutional neural network ${ }^{69}$ \\
\hline Noonan syndrome & DeepGestalt technology ${ }^{33}$ \\
\hline
\end{tabular}


Table 2 Example PubMed search strategy

\begin{tabular}{ll}
\hline Concept & Search string \\
\hline Deep learning & "deep learning"[MeSH] OR "deep learning"[TIAB] OR "artificial intelligence" [MeSH] OR "artificial \\
& intelligence" [TIAB] OR "machine learning"[MeSH] OR "machine learning"[TIAB] OR "convolutional \\
& neural network"[MeSH] OR "convolutional neural network"[TIAB] OR "convolutional neural \\
& networks"[TIAB]
\end{tabular}

systematic review on the state-of-the-art applications of deep learning-based facial analysis in non-invasively evaluating medical conditions. Therefore, to bridge this gap, we aim to systematically review the literature and identify the characteristics and effects of deep learning-based facial analysis techniques applied in medical research.

\section{METHODS AND ANALYSIS}

This systematic review was registered with the International Prospective Register of Systematic Reviews database or PROSPERO a priori to improve research rigour. ${ }^{3738}$ The principles of the Preferred Reporting Items for Systematic Reviews and Meta-Analyses protocol was adopted to guide this systematic review. ${ }^{39}$ Our search strategy incorporated medical subject heading $(\mathrm{MeSH})$ and keyword terms for the concept of deep learning and facial analysis. The search strategy was developed in consultation with an academic librarian, and subsequently will be deployed to target databases, including PubMed, PsycINFO, CINAHL, IEEEXplore and Scopus (table 2). The search will be initiated in September 2021. Studies will be limited to journal articles published in English. We will adopt two additional search mechanisms to locate eligible articles: (1) a manual search of the reference list of the included articles will be performed and (2) a reverse search of papers that cited articles included in the final review via Google Scholar. An academic librarian will facilitate the search process, helping administer the search and download the citation records to Rayyan (http://rayyan.qcri.org).

\section{Inclusion and exclusion criteria}

The inclusion criteria were developed a priori and listed in table 3. Studies will be excluded articles if they (1) did not report findings on human beings (eg, studies on mice), (2) did not focus on full facial features (eg, research on retina or lip-cleft), (3) did not conduct research in a medical context (eg, in the context of criminology) and (4) did not report empirical findings (eg, editorial or comment papers).

\section{Risk of bias assessment}

To ensure the quality of included studies, a risk of bias assessment will be conducted independently by two reviewers, using the Cochrane Collaboration evaluation framework. ${ }^{40}$ The framework has seven domains: (1) random sequence generation, (2) allocation concealment, (3) blinding of participants and personnel, (4) blinding of outcome assessment, (5) incomplete outcome data, (6) selective reporting and (7) any other source of bias. The risk of bias will be evaluated independently by two reviewers. Potential discrepancies regarding the risk of bias will be resolved via group discussions till a consensus is reached.

\section{Data extraction}

Two reviewers will independently examine the citations and select studies for inclusion. Discrepancies will be resolved by group discussions till a consensus is reached. Data will be extracted based on the research objective and selection criteria adopted in this study. For articles that meet the inclusion criteria, the reviewers will extract the following information from the included papers: research objective/questions, disease context, sample characteristics (eg, characteristics of facial records), AI characteristics (eg, algorithm adopted), and empirical findings.

\section{Data synthesis and analysis}

If eligible studies share enough similarities to be pooled, a meta-analysis will be conducted to gain further insights into the data. Main clinical, methodological, as well as statistical differences will be carefully considered to determine the heterogeneity of the eligible studies. If eligible studies are found heterogeneous, a narrative synthesis will

\begin{tabular}{|c|c|}
\hline Data type & Inclusion criteria \\
\hline Participants & $\begin{array}{l}\text { Individuals younger or older than } 18 \\
\text { years old }\end{array}$ \\
\hline Research context & Medical research or healthcare \\
\hline Analytical technique & $\begin{array}{l}\text { Deep learning algorithms-based facial } \\
\text { image analysis }\end{array}$ \\
\hline Language & English \\
\hline Study type & Quantitative empirical study \\
\hline Outcome & $\begin{array}{l}\text { Report empirical and original findings } \\
\text { on the application of deep learning- } \\
\text { based facial image analysis in medical } \\
\text { context (eg, accuracy of facial image } \\
\text { analysis in detecting Down syndrome) }\end{array}$ \\
\hline
\end{tabular}




\begin{tabular}{|c|c|}
\hline Cause & Definition and example \\
\hline Gene-related factors & $\begin{array}{l}\text { Gene-related factors are causes for individuals' abnormal facial changes that root in the } \\
\text { presence or mutation of one or a set of genes. } \\
\text { Examples: Down syndrome (genetic root: presence of a third copy of chromosome } 21 \text { ) or } \\
\text { Cornelia de Lange syndrome (genetic root: NIPBL or SMC1A, SMC3, RAD21 or HDAC8, } \\
\text { BRD4 and ANKRD11 genes). }{ }^{5414255}\end{array}$ \\
\hline Medication-induced triggers & $\begin{array}{l}\text { Medication-induced triggers could be understood as causes to individuals' short-term or } \\
\text { long-term abnormal facial changes due to their adverse reactions to a certain medication of } \\
\text { a type of medications. } \\
\text { Examples: Neuroleptic malignant syndrome (antipsychotic drugs), tardive dyskinesia } \\
\text { (antipsychotic medications) or drug-related Tourette syndrome. }{ }^{51-54}\end{array}$ \\
\hline
\end{tabular}

be conducted to summarise the data. A summary of the data extracted will be organised to synthesise key results. Both tables and graphs will be used to represent the key characteristics of eligible articles. Descriptive analysis will be performed on categorical variables. In this review, we will undertake a narrative approach to synthesise data. In other words, in addition to shedding light on key information like the sensitivity, specificity, overall accuracy of the deep learning technologies in analysing facial images (as opposed to clinicians' analyses), we will also provide detailed analysis of the disease contexts and the techniques applied to chart the state-of-the-art of deep learning technologies in facial image analyses.

\section{Ethics and dissemination}

As the study is a protocol for a systematic review, ethical approval is not required. The study findings will be disseminated via peer-reviewed publications and conference presentations.

\section{Patient and public involvement}

The nature of the study, which is a review and analysis of previously published data, dictates that there is limited to no meaningful need for patient and public involvement in the design, delivery or dissemination of the research findings.

\section{DISCUSSION}

Though a growing body of research has applied deep learning-based facial image analysis in the medical context for disease detection, diagnosis and prognosis, to date, no systematic review has investigated the stateof-the-art application of deep learning-based facial image analysis recognition in addressing medical diagnoses and clinical states. Therefore, to bridge this gap, we aim to systematically review the literature and present the characteristics, challenges, as well as opportunities in deep learning-based facial analysis techniques applied in medical research. To better organise the research findings, we developed a framework that illustrates the main causes for abnormal facial expressions in patients. It is important to note that we are identifying medical states and conditions and not individuals.

After reviewing the literature,$^{541-55}$ we identified the following four preliminary categories of causes for shortterm or long-term abnormal facial expressions in people: (1) gene-related factors, (2) neurological factors, (3) psychiatric conditions and (4) medication-induced triggers. Genetic-related factors, such as the presence or mutation of a certain gene, are the most studied cause for abnormal facial changes in individuals. ${ }^{5} 75$ Down syndrome, which is affected by the presence of a third copy of chromosome 21, is an example of genetic-related factors that can cause individuals' abnormal facial changes. ${ }^{5}$ Neurological factors can also cause individuals' facial phenotypes. Stroke or transient ischaemic attack is an example of neurological factors, which can occur either prior to or after the onset of the disease. ${ }^{567}$ The third cause for abnormal facial changes centres on individuals' psychiatric conditions or mental illnesses, especially 
psychotic disorders such as the Tourette syndrome (facial tics). Last but not the least, medication-induced triggers, such as the neuroleptic malignant syndrome (caused by antipsychotic medications), can also cause abnormal facial changes in people. Details of this framework can be found in table 4 . This framework will be used in the planned systematic review study to guide the data extraction process.

Overall, insights gained from this study will be able to provide a much-needed understanding of the characteristics, challenges, as well as opportunities in the context of deep learning-based facial image analysis technologies applied in disease detection, diagnosis and prognosis. In addition to gaining a connected and comprehensive understanding of the current application of facial image analysis, results of the study will also be able to shed light on whether, similar to facial recognition used in nonmedical ${ }^{58} 59$ and medical contexts ${ }^{6061}$ whether or to what degree is systematic bias is present in the application of deep learning technologies for facial image analysis. A biased and inaccurate facial image analysis system will not only exert unwarranted, though avoidable, disparities on patients (eg, gender inequality) ${ }^{60}$ it will also alienate the patients from the much-needed deep-learning-assisted medical opportunities their health and well-being can benefit from. ${ }^{62}$ Therefore, for the consideration of patients' welfare and the development of the clinical practice, a timely understanding of the scope of the research literature as well as the challenges and opportunities faced by research on deep-learning-based facial image analysis is much needed.

\section{Author affiliations}

${ }^{1}$ Center on Smart and Connected Health Technologies, Mays Cancer Center, School of Nursing, UT Health San Antonio, San Antonio, Texas, USA

${ }^{2}$ Department of Radiation Oncology, Chinese Academy of Medical Sciences and Peking Union Medical College, Beijing, China

${ }^{3}$ Department of Research and Development, Shanghai United Imaging Intelligence Co., Ltd, Shanghai, China

${ }^{4}$ Epidemiology and Biostatistics, University of Texas Health Science Center at San Antonio, San Antonio, UK

${ }^{5}$ Department of Microbiology, University of Sarajevo, Sarajevo, Bosnia and Herzegovina

${ }^{6}$ College of Nursing, Florida State University, Tallahassee, Florida, USA

${ }^{7}$ School of Resource and Environmental Sciences, Wuhan University, Wuhan, China ${ }^{8}$ International Institute of Spatial Lifecourse Epidemiology (ISLE), Wuhan University, Wuhan, China

${ }^{9}$ Division of Health Security Research, National Health Commission of the People's Republic of China, Beijing, Beijing, China

Correction notice This article has been corrected since it first published. Affiliation for 'Peng Jia' has been updated.

Acknowledgements The authors wish to thank Emme Lopez, for her assistance in the search strategy development. Furthermore, the authors are very grateful for the constructive input offered by the editors and reviewers.

Contributors ZS developed the research idea and drafted the manuscript. BL, FS, JG, SS, JW, PJ and XH reviewed and revised the manuscript.

Funding This work was supported by the United Nations Development Program (UNDP) South-South Cooperation: Learning from China's Experience to improve the Ability of Response to COVID-19 in Asia and the Pacific Region; The Joint Pilot Project between the Ministry of Industry and Information Technology and the National Health Commission of the People's Republic of China: The Development,
Standardization, and Application of 5G-Powered and Cloud-Based Virtual Critical Care and Management.

Competing interests None declared.

Patient and public involvement Patients and/or the public were not involved in the design, or conduct, or reporting, or dissemination plans of this research.

Patient consent for publication Not applicable.

Provenance and peer review Not commissioned; externally peer reviewed.

Open access This is an open access article distributed in accordance with the Creative Commons Attribution Non Commercial (CC BY-NC 4.0) license, which permits others to distribute, remix, adapt, build upon this work non-commercially, and license their derivative works on different terms, provided the original work is properly cited, appropriate credit is given, any changes made indicated, and the use is non-commercial. See: http://creativecommons.org/licenses/by-nc/4.0/.

ORCID iDs

Zhaohui Su http://orcid.org/0000-0003-2005-9504

Sabina Šegalo http://orcid.org/0000-0002-9280-3278

\section{REFERENCES}

1 Yang J, Zhang K, Fan $\mathrm{H}$, et al. Development and validation of deep learning algorithms for scoliosis screening using back images. Commun Biol 2019;2): :390.

2 Wang X, Liu J, Wu C, et al. Artificial intelligence in tongue diagnosis: using deep convolutional neural network for recognizing unhealthy tongue with tooth-mark. Comput Struct Biotechnol J 2020;18:973-80.

3 Lam C, Yi D, Guo M, et al. Automated detection of diabetic retinopathy using deep learning. AMIA Jt Summits Trans/ Sci Proc 2018;2017:147-55.

4 Martin D, Croft J, Pitt A, et al. Systematic review and meta-analysis of the relationship between genetic risk for schizophrenia and facial emotion recognition. Schizophr Res 2020;218:7-13.

5 Kruszka P, Porras AR, Sobering AK, et al. Down syndrome in diverse populations. Am J Med Genet A 2017;173:42-53.

6 Liang B, Yang N, He G, et al. Identification of the facial features of patients with cancer: a deep learning-based pilot study. J Med Internet Res 2020;22:e17234.

7 Latorre-Pellicer A, Ascaso Ángela, Trujillano L, et al. Evaluating face2gene as a tool to identify cornelia de lange syndrome by facial phenotypes. Int J Mol Sci 2020;21. doi:10.3390/ijms21031042. [Epub ahead of print: $04 \mathrm{Feb} 2020]$.

8 Myers L, Anderlid B-M, Nordgren A, et al. Clinical versus automated assessments of morphological variants in twins with and without neurodevelopmental disorders. Am J Med Genet A 2020;182:1177-89.

9 Deng L, Yu D. Deep learning: methods and applications. FNT in Signal Processing 2013;7:197-387.

10 LeCun Y, Bengio Y, Hinton G. Deep learning. Nature 2015;521:436-44.

11 Gilani SZ, Mian A. Learning from millions of 3D scans for large-scale 3D face recognition. IEEE/CVF Conference on Computer Vision and Pattern Recognition, 2018.

12 Algabri R, Choi M-T. Deep-learning-based indoor human following of mobile robot using color feature. Sensors 2020;20. doi:10.3390/ s20092699. [Epub ahead of print: 09 May 2020].

13 Yin J, Apuroop KGS, Tamilselvam YK, et al. Table cleaning task by human support robot using deep learning technique. Sensors 2020;20:1698.

14 Balado J, Martínez-Sánchez J, Arias P, et al. Road environment semantic segmentation with deep learning from MLS point cloud data. Sensors 2019;19:3466.

15 Naqvi RA, Arsalan M, Batchuluun G, et al. Deep learning-based gaze detection system for automobile drivers using a NIR camera sensor. Sensors 2018;18:456.

16 Liu G, Hua J, Wu Z, et al. Automatic classification of esophageal lesions in endoscopic images using a convolutional neural network. Ann Transl Med 2020;8:486.

17 Chartrand G, Cheng PM, Vorontsov E, et al. Deep learning: a primer for radiologists. Radiographics 2017;37:2113-31.

18 Jeong Y, Kim JH, Chae H-D, et al. Deep learning-based decision support system for the diagnosis of neoplastic gallbladder polyps on ultrasonography: preliminary results. Sci Rep 2020;10:7700.

19 Sánchez Fernández I, Yang E, Calvachi P, et al. Deep learning in rare disease. detection of tubers in tuberous sclerosis complex. PLOS One 2020;15:e0232376. 
20 Marro A, Bandukwala T, Mak W. Three-dimensional printing and medical imaging: a review of the methods and applications. Curr Probl Diagn Radiol 2016;45:2-9.

21 Atutornu J, Hayre CM. Personalised medicine and medical imaging: opportunities and challenges for contemporary health care. $J$ Med Imaging Radiat Sci 2018;49:352-9.

22 Afshar P, Mohammadi A, Plataniotis KN, et al. From handcrafted to deep-learning-based cancer radiomics: challenges and opportunities. IEEE Signal Process Mag 2019;36:132-60.

23 Antropova N, Huynh BQ, Giger ML. A deep feature fusion methodology for breast cancer diagnosis demonstrated on three imaging modality datasets. Med Phys 2017;44:5162-71.

24 Giger ML. Machine learning in medical imaging. J Am Coll Radiol 2018;15:512-20.

25 Sahiner B, Pezeshk A, Hadjiiski LM, et al. Deep learning in medical imaging and radiation therapy. Med Phys 2019;46:e1-36.

26 Huang S, Yang J, Fong S, et al. Artificial intelligence in cancer diagnosis and prognosis: opportunities and challenges. Cancer Lett 2020;471:61-71.

27 Litjens G, Sánchez Cl, Timofeeva N, et al. Deep learning as a tool for increased accuracy and efficiency of histopathological diagnosis. Sci Rep 2016;6:26286.

28 Moitra D, Mandal RK. Prediction of non-small cell lung cancer histology by a deep ensemble of convolutional and bidirectiona recurrent neural network. J Digit Imaging 2020;33:895-902.

29 Mahbod A, Schaefer G, Wang C, et al. Transfer learning using a multi-scale and multi-network ensemble for skin lesion classification. Comput Methods Programs Biomed 2020;193:105475.

30 Li Z, Togo R, Ogawa T, et al. Chronic gastritis classification using gastric X-ray images with a semi-supervised learning method based on tri-training. Med Biol Eng Comput 2020;58:1239-50.

31 Liu S, Shah Z, Sav A, et al. Isocitrate dehydrogenase (IDH) status prediction in histopathology images of gliomas using deep learning. Sci Rep 2020:10:7733.

32 Kim YD, Noh KJ, Byun SJ, et al. Effects of hypertension, diabetes, and smoking on age and sex prediction from retinal fundus images. Sci Rep 2020:10:4623

33 Gurovich Y, Hanani Y, Bar O, et al. Identifying facial phenotypes of genetic disorders using deep learning. Nat Med 2019;25:60-4.

34 Wang $\mathrm{Y}$, Kosinski M. Deep neural networks are more accurate than humans at detecting sexual orientation from facial images. $J$ Pers Soc Psychol 2018:114:246-57.

35 Kruszka P, Addissie YA, McGinn DE, et al. 22Q11.2 deletion syndrome in diverse populations. Am J Med Genet A 2017:173:879-88.

36 Elmas M, Gogus B. Success of face analysis technology in rare genetic diseases diagnosed by whole-exome sequencing: a singlecenter experience. Mol Syndromol 2020;11:4-14.

$37 \mathrm{Xu}$ C, Cheng L-L, Liu Y, et al. Protocol registration or development may benefit the design, conduct and reporting of dose-response meta-analysis: empirical evidence from a literature survey. BMC Med Res Methodol 2019;19:78.

38 Dos Santos MBF, Agostini BA, Bassani R, et al. Protocol registration improves reporting quality of systematic reviews in dentistry. $B M C$ Med Res Methodol 2020;20:57.

39 Moher D, Shamseer L, Clarke M, et al. Preferred reporting items for systematic review and meta-analysis protocols (PRISMA-P) 2015 statement. Syst Rev 2015;4:1.

40 Higgins JPet al. Cochrane handbook for systematic reviews of interventions. John Wiley \& Sons, 2019.

41 Basel-Vanagaite L, Wolf L, Orin M, et al. Recognition of the cornelia de Lange syndrome phenotype with facial dysmorphology novel analysis. Clin Genet 2016;89:557-63.

42 Cha S, Lim JE, Park AY, et al. Identification of five novel genetic loci related to facial morphology by genome-wide association studies. BMC Genomics 2018;19:481.

43 Richmond S, Howe LJ, Lewis S, et al. Facial genetics: a brief overview. Front Genet 2018;9:462.

44 Spencer CR, Irving RM. Causes and management of facial nerve palsy. Br J Hosp Med 2016;77:686-91.

45 Fasano A, Tinazzi M. Functional facial and tongue movement disorders. In: Handbook of clinical neurology. Elsevier, 2016: 353-65.

46 Hagberg B. Clinical manifestations and stages of Rett syndrome. Ment Retard Dev Disabil Res Rev 2002;8:61-5.
47 Tan N-C, Chan L-L, Tan E-K. Hemifacial spasm and involuntary facial movements. QJM 2002;95:493-500.

48 Liu T-L, Wang P-W, Yang Y-HC, et al. Association between facial emotion recognition and bullying involvement among adolescents with high-functioning autism spectrum disorder. Int J Environ Res Public Health 2019;16:5125.

49 Boutrus M, Gilani SZ, Alvares GA, et al. Increased facial asymmetry in autism spectrum conditions is associated with symptom presentation. Autism Res 2019;12:1774-83.

50 Gill CE, Kompoliti K. Clinical features of tourette syndrome. J Child Neurol 2020;35:166-74

51 Kim DD, Barr AM, Chung Y, et al. Antipsychotic-associated symptoms of tourette syndrome: a systematic review. CNS Drugs 2018;32:917-38

52 Thomas CS. A study of facial dysmorphophobia. Psychiatr Bull 1995;19:736-9.

53 Phillips KA, Cash TF, Smolak L. Body image and body dysmorphic disorder eating disorders and obesity: a comprehensive Handbook. , 2002: 2, 115.

54 Reese HE, McNally RJ, Wilhelm S. Facial asymmetry detection in patients with body dysmorphic disorder. Behav Res Ther 2010;48:936-40.

55 White JJ, Mazzeu JF, Hoischen A, et al. DVL3 alleles resulting in a -1 frameshift of the last exon mediate autosomal-dominant robinow syndrome. Am J Hum Genet 2016;98:553-61.

56 Onder H, Albayrak L, Polat H. Frontal lobe ischemic stroke presenting with peripheral type facial palsy: a crucial diagnostic challenge in emergency practice. Turk J Emerg Med 2017;17:112-4.

57 Han Y-Y, Qi D, Chen X-D, et al. Limb-shaking transient ischemic attack with facial muscles involuntary twitch successfully treated with internal carotid artery stenting. Brain Behav 2020;10:e01679.

58 Buolamwini J, Gebru T. Gender shades: Intersectional accuracy disparities in commercial gender classification. In: Proceedings of the 1st conference on Fairness, accountability and transparency, A.F. PMLR: Proceedings of Machine Learning Research, 2018: 77-91.

59 Drozdowski P, Rathgeb C, Dantcheva A, et al. Demographic bias in biometrics: a survey on an emerging challenge. IEEE Transactions on Technology and Society 2020;1:89-103.

60 Parikh RB, Teeple S, Navathe AS. Addressing bias in artificial intelligence in health care. JAMA 2019;322:2377-8.

61 Obermeyer Z, Powers B, Vogeli C, et al. Dissecting racial bias in an algorithm used to manage the health of populations. Science 2019;366:447-53.

62 Garcia RV. The harms of demographic bias in feep face recognition research. International Conference on Biometrics (ICB), 2019.

63 Kong X, Gong S, Su L, et al. Automatic detection of acromegaly from facial photographs using machine learning methods. EBioMedicine 2018;27:94-102.

64 Liang B, Yang N, He G, et al. Identification of the facial features of patients with cancer: a deep learning-based pilot study. $J$ Med Internet Res 2020;22:e17234.

65 Lin S, Li Z, Fu B, et al. Feasibility of using deep learning to detect coronary artery disease based on facial photo. Eur Heart $J$ 2020;41:4400-11.

66 Zhao Q, Okada K, Rosenbaum K, et al. Digital facial dysmorphology for genetic screening: hierarchical constrained local model using ICA. Med Image Anal 2014;18:699-710.

67 Goceri E. Deep learning based classification of facial dermatological disorders. Comput Biol Med 2021;128:104118.

68 Han SS, Moon IJ, Lim W, et al. Keratinocytic skin cancer detection on the face using region-based convolutional neural network. JAMA Dermatol 2020;156:29-37.

69 Camino A, Wang Z, Wang J, et al. Deep learning for the segmentation of preserved photoreceptors on en face optical coherence tomography in two inherited retinal diseases. Biomed Opt Express 2018;9:3092-105.

70 Bargshady G, Zhou X, Deo RC, et al. Enhanced deep learning algorithm development to detect pain intensity from facial expression images. Expert Syst Appl 2020;149:113305.

71 Yolcu G. Deep learning-based facial expression recognition for monitoring neurological disorders. IEEE International Conference on Bioinformatics and Biomedicine (BIBM), 2017. 